\title{
Analysis of Synchronous Conditions in the Cylindrical Magnetron Space Charge*
}

\author{
H. W. Welch, JR., aNd W. G. Dow \\ Engineering Research Institute, University of Michigan, Ann Arbor, Michigan
}

(Received August 31, 1950)

\begin{abstract}
In the multianode cylindrical magnetron there exist favored phase velocities of the electromagnetic wave around the interaction space between anode and cathode. These velocities are characteristic of the resonant system attached to the anode segments. In the oscillating magnetron the electronic space charge within the interaction space is presumed to maintain sychronism with one of these velocities. Certain of the conditions of synchronism which can be discussed analytically are treated in this paper. The restilts, although based on restrictive assumptions, can be used in the interpretation of magnetron operation and in predicting regions of efficient behavior.
\end{abstract}

\section{INTRODUCTION}

$T$ $\mathrm{HE}$ analysis of space charge distribution in the static magnetron diode has been discussed in a number of papers ${ }^{1}$ and has admittedly only been treated under very special assumptions. In spite of this the results can be very useful in many practical applications if properly handled. The resulting picture is essentially that of a swarm of electrons moving around the cathode having a radial extent determined by the applied dc anode voltage and axial magnetic field.

This paper discusses a similar solution for a magnetron in which an rf voltage is assumed present between anode segments uniformly spaced about the magnetron anode periphery. A voltage of this type varying in time has been considered by Slater and others as the resultant of a number of $\mathrm{rf}$ waves traveling in opposite directions around the magnetron periphery causing the time varying fields to exist in the space between anode and cathode. This space is called the interaction space because it is the space common to the rf fields and the electrons where interaction takes place. Only one type of wave is considered of importance in the present discussion. This is a wave traveling in the same direction as the electrons moving around the interaction space so that interaction between the electrons and the wave is appreciable. Waves traveling in the opposite direction are considered as contributing only to higher order corrections.

\section{EQUATIONS GOVERNING SPACE CHARGE BEHAVIOR}

The mathematical analysis of space charge behavior in a magnetron is based on the force equation for an electron in an electromagnetic field. This equation in vector form is the following

$$
d \mathbf{v} / d t=-e / m(\mathbf{F}+\mathbf{v} \times \mathbf{B}),
$$

where $e=$ absolute value of electronic charge, $m=$ mass

* The material in this paper is based on work done for the U. S. Army Signal Corps on Contract No. W36-039 sc-35561.

${ }^{1}$ L. Brillouin, Phys. Rev. 60, 385-396 (1941). J. P. Blewett and S. Ramo, Phys. Rev. 57, 635-641 (1940). J. C. Slater, Microwave Electronics (D. Van Nostrand Company, Inc., New York, 1950), Chapter VIII. L. Page and N. I. Adams, Phys. Rev. 69, 492 (1946). G. B. Collins, Microwave Magnetrons (McGraw-Hill Book Company, Inc., New York, 1948), Part II. of electron, $\mathbf{v}=$ velocity of electron, $\mathbf{F}=$ vector value of electric field, $\mathbf{B}=$ vector value of magnetic field.

The magnetron will be considered with axial alignment in the z-direction. In this case it is convenient to resolve the vector quantities in Eq. (1) into components in cylindrical coordinates. If proper account is taken of the symmetries in the magnetron, Eq. (1) may be written as follows:

$$
\begin{aligned}
\frac{d \mathbf{v}}{d t} & =\mathbf{r}_{1}\left\{\frac{d v_{r}}{d t}-r \omega^{2}\right\}+\boldsymbol{\theta}_{1}\left\{2 v_{r} \omega+r \frac{d \omega}{d t}\right\} \\
& =-e / m\left(\mathbf{r}_{1} F_{r}+\boldsymbol{\theta}_{1} F_{\theta}+\mathbf{r}_{1} r \omega B_{z}-\boldsymbol{\theta}_{1} v_{r} B_{z}\right) .
\end{aligned}
$$

Here the quantities with subscript (1) are unit vectors. $\mathbf{v}$ has been replaced by

$$
\mathbf{v}=\mathbf{r}_{1} v_{r}+\boldsymbol{\theta}_{1} r \omega
$$

$r \omega$ is therefore the magnitude of the $\theta$-directed component of velocity. $\omega$ is the angular velucity of the electron. The components $F_{z}, v_{z}, B_{\theta}, B_{r}$ are considered non-existent as would be the case in an ideal magnetron. By separation of the $r$ - and $\theta$-directed components in Eq. (1a) we obtain the following two important equations for scalar quantities.

$$
\begin{aligned}
d v_{r} / d l-r \omega^{2} & =-e / m\left(F_{r}+r \omega B_{z}\right), \\
2 v_{r} \omega+r d \omega / d t & =-e / m\left(F_{\theta}-v_{r} B_{z}\right) .
\end{aligned}
$$

There are several results to be derived from these two equations based on various assumptions which may be used. We will consider two cases of interest. Since $B$ is always in the $z$-direction we will drop the subscript $z$.

\section{THE STATIC MAGNETRON, NO If VOLTAGE PRESENT}

This case will be discussed very briefly to give the necessary results, since it has been treated in the references.

We will first assume that no rf field is present; therefore, $F_{\theta}=0$. In this case Eq. (3b) can be written

$$
2 d r / d t\left(\omega_{L}-\omega\right)=r d \omega / d t .
$$

$v_{r}$ has been replaced by $d r / d t$ and $e B / 2 m$ has been replaced by $\omega_{L}$. The equation is readily solved by separa- 
tion of variables and assuming $\omega$ to be zero at the boundary of the cathode $r_{c}$.

$$
\omega=\omega_{L}\left[1-\left(r_{c}^{2} / r^{2}\right)\right] .
$$

This result may be substituted back into Eq. (3a) yielding an equation which can be solved simply only under the restrictive assumption that

Now,

$$
d v_{r} / d t=0 .
$$

$$
d v_{r} / d t=\partial v_{r} / \partial t+\left(\partial v_{r} / \partial r\right) v_{r}
$$

therefore, in order for $d v_{r} / d t$ to be zero,

$$
\partial v_{r} / \partial t=0
$$

implying a steady-state condition, and

$$
v_{r}=0
$$

implying that no radial current exists.

The potential distribution resulting in this case is actually obvious from Eq. (5) and the assumptions just stated

$$
E-E_{c}=1(m / 2 e) \omega_{L}^{2} r^{2}\left[1-\left(r_{c}^{2} / r^{2}\right)\right]^{2}
$$

where $E=$ potential at $r$ and $E_{c}=$ potential at $r_{c}$. This relationship states simply that the energy taken by the electron from the electric field is equal to its kinetic energy. Using the poisson equation the space charge density under these conditions can be shown to be

$$
\rho / \epsilon_{0}=-2 m / e \omega_{L}^{2}\left[1+\left(r_{c}^{4} / r^{4}\right)\right]
$$

where $\epsilon_{0}=$ dielectric constant of free space $=1 / 36 \pi \times 10^{-9}$ farad $/ \mathrm{m}$. The electric field is given by

$$
F_{r}=-\partial E / \partial r=(m / e) r \omega_{L}^{2}\left[\left(r_{c}^{4} / r^{4}\right)-1\right] .
$$

It is important to realize that Eqs. (7)-(9) represent distributions within the space charge swarm only for the special case of no radial current throughout the swarm. Equations (7) and (9) do hold more significance for the swarm boundary. Such a boundary must exist if the anode potential is less than the value necessary to make the swarm extend all the way out to the anode. The potential is given by substituting the anode radius $\left(r_{a}\right)$ into Eq. (7). [The electron must have at leasi the energy corresponding to the angular velocity of Eq. (5) since this result is independent of the solution of Eq. (3a).] We are led to the conclusion that if the swarm is bounded within the interaction space between anode and cathode no current is crossing the boundary, therefore Eqs. (7) and (9) must hold at the boundary. By the gaussian theorem the total space charge per unit length within a boundary at radius $r_{H}$ will, therefore, be

$$
\begin{aligned}
\tau_{H} & =2 \pi \epsilon_{0}[-(\partial E / \partial r)] \text { at } r_{H} \\
& =(1 \pi / 2) \epsilon_{0}(e / m) B^{2} r_{H}{ }^{2}\left[\left(r_{c}^{2} / r_{H}{ }^{2}\right)-1\right] .
\end{aligned}
$$

We must conclude that the total space charge within a bounded swarm is independent of the potential, field and space charge distribution within the swarm. Electrons may be circulating around the cathode or stream- ing radially outward and inward through the swarm provided they do not cross the boundary $r_{H}$. If there are radial velocities within the swarm the potential within the swarm must be greater than that given by Eq. (7).

The purpose of this discussion is to emphasize the fact that the relationship for the radius of the swarm boundary in terms of anode potential which follows is not dependent on the restrictive assumptions corresponding to the existence of the distributions of Eqs. (7)-(9) throughout the swarm. It is only dependent on the assumptions that the initial velocity and the radial velocity are zero and that no sources of energy exist other than the dc field.

If we consider the magnetron as a cylindrical diode containing the space charge swarm just described and having no gradient at the cathode then

$$
E_{a}-E_{H}=-\left(\tau_{H} / 2 \pi \epsilon_{0}\right) \log \left(r_{a} / r_{H}\right),
$$

where $E_{a}=$ anode potential and $E_{H}=$ value given by Eq. (7) for $r_{H}$. We have the complete result

$$
\begin{aligned}
& E_{a}=B^{2} \frac{e}{8 m} r_{H}^{2}\left[2\left(1-\frac{r_{c}^{4}}{r_{H}^{4}}\right) \frac{r_{a}}{r_{H}}+\left(1-\frac{r_{c}^{2}}{r_{H}^{2}}\right)^{2}\right] . \\
& \text { CONDITIONS FOR SYNCHRONISM WITH THE If } \\
& \text { FIELD IN THE MAGNETRON }
\end{aligned}
$$

In the discussion of this section it is not actually necessary for an rf field to be present. It is only necessary to define the angular velocity around the interaction space which corresponds to a particular rf field which might be present.

It will be convenient to define some terms which will simplify the following discussion and will make the terminology the same as that generally used in discussing the scaling of magnetrons. ${ }^{2}$ These definitions also have the advantage of making most of the equations dimensionless. The presence of the $\mathrm{rf}$ is described in the usual manner. ${ }^{2}$ The rf wave with which the electrons interact is considered as traveling around the interaction space between anode and cathode, with an angular velocity given by

$$
\omega_{n}=\frac{2 \pi f}{n} \text { radians } / \mathrm{sec}
$$

where $f$ is the frequency of the rf impressed on the magnetron anodes and $n$ is the mode number, equal to $N / 2$ in the $\pi$-mode where $N$ is the number of anodes.

The kinetic energy of an electron at the anode having the angular velocity given by Eq. (12) defines a voltage which we will call $E_{0}$

$$
E_{0}=(m / 2 e) \omega_{n}{ }^{2} r_{a}{ }^{2} .
$$

The magnetic field which would allow this angular velocity to exist at the anode of a non-oscillating magnetron is defined by Eq. (5) when $\omega$ is equal to $\omega_{n}$

\footnotetext{
${ }^{2}$ See reference 1, J. C. Slater, Chapter 13.
} 
and $r$ set equal to $r_{a}$

$$
B_{0}=\frac{2 m}{e} \omega_{n} \frac{1}{\left[1-\left(r_{c}^{2} / r_{a}^{2}\right)\right]} .
$$

In terms of these variables Eq. (7) may be written

$$
\left(E-E_{c}\right) / E_{0}=\left(B / B_{0}\right)^{2} \text {. }
$$

$E_{0}$ and $B_{0}$ depend only on dimensions of the magnetron, the frequency impressed on the magnetron anodes and physical constants.

At a particular voltage, outer electrons in the swarm reach the angular velocity of the rf field given by Eq. (12). This we will call the synchronous voltage. In order to get an idea of the radius of the space charge for which synchronism takes place, it is convenient to rewrite Eq. (11) in terms of the new variables as follows:

$$
\begin{array}{r}
\frac{E_{a}}{E_{0}}=\left(\frac{B}{B_{0}}\right)^{2}\left(\frac{r_{H}}{r_{a}}\right)^{2}\left\{2 \frac{1-\left(r_{c}^{4} / r_{H}^{4}\right)}{\left[1-\left(r_{c}^{2} / r_{a}^{2}\right)\right]^{2}} \ln \frac{r_{a}}{r_{H}}\right. \\
\left.+\left[\frac{1-\left(r_{c}^{2} / r_{H}{ }^{2}\right)}{1-\left(r_{c}^{2} / r_{a}^{2}\right)}\right]^{2}\right\} .
\end{array}
$$

Using the definition of $B_{0}$ we write

When $\omega=\omega_{n}$

$$
\frac{\omega}{\omega_{n}}=\frac{B}{B_{0}} \frac{1-\left(r_{c}^{2} / r^{2}\right)}{1-\left(r_{c}^{2} / r_{a}^{2}\right)} .
$$

$$
\frac{B_{n}}{B_{0}}=\frac{1-\left(r_{c}^{2} / r_{a}^{2}\right)}{1-\left(r_{c}^{2} / r_{n}^{2}\right)}
$$

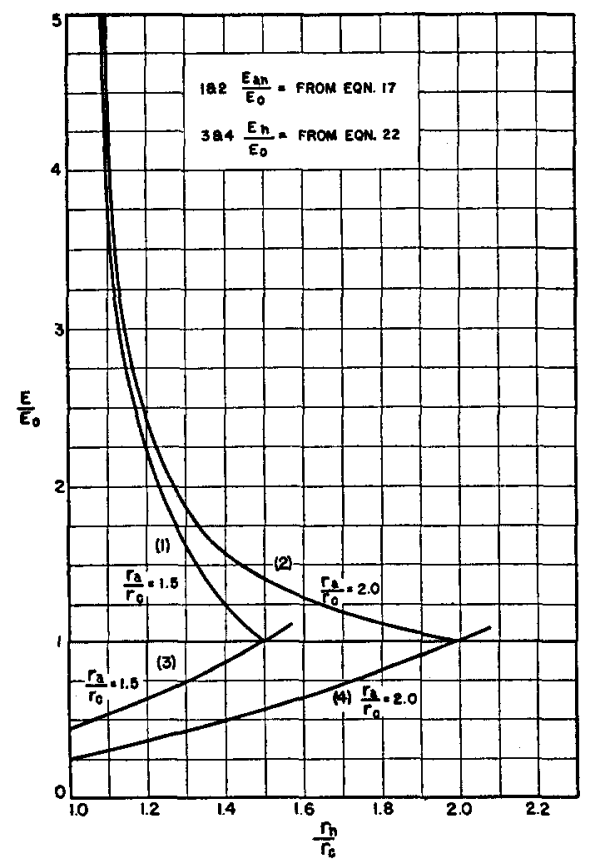

Fig. 1. Anode voltage and space charge cloud boundary voltage as function of radius of space charge cloud boundary for synchronism of outer electrons.

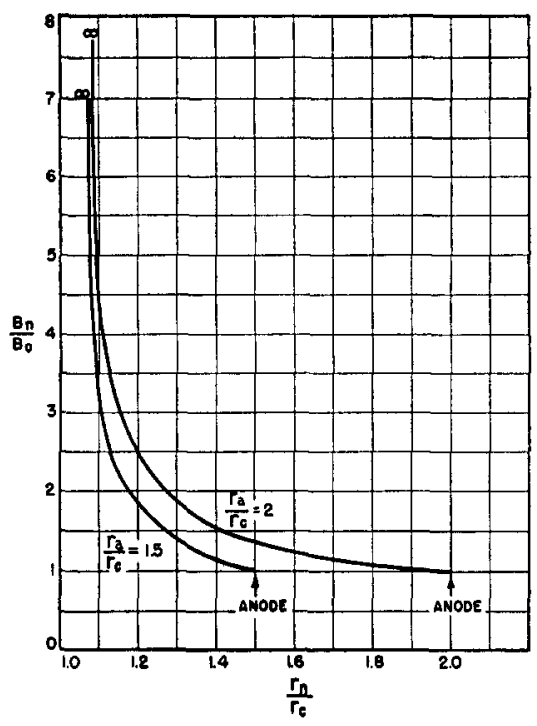

FIG. 2. Magnetic field as function of radius of space charge cloud boundary for synchronism of outer electrons. [From Eq. (16).]

The subscript $n$ denotes values for which the angular velocity of the outer edge of the electron swarm reaches synchronism with the angular velocity of the wave. The voltage for which synchronism occurs at a given radius is given by substitution of $B_{n} / B_{0}$ from Eq. (16) into Eq. (11). We are discussing the outer edge of the cloud so $r_{H}=r_{n}$.

$$
\frac{E_{a n}}{E_{0}}=\frac{r_{n}^{2}}{r_{a}^{2}}\left[2 \frac{1+\left(r_{c}^{2} / r_{n}^{2}\right)}{1-\left(r_{c}^{2} / r_{n}^{2}\right)} \ln \frac{r_{a}}{r_{n}}+1\right] .
$$

An interesting conclusion to be drawn from Eqs. (16) and (17) is that for given values of $\omega_{n}$ and $r_{n}$ and a given tube geometry there is only one set of values for $E_{a} / E_{0}$ and $B / B_{0}$, which will allow the edge of the electron swarm to rotate in synchronism. Equations (16) and (17) are plotted in Figs. 1 and 2 for two values of $r_{a} / r_{c}$.

By eliminating $r_{n}$ between these two equations, we may write an equation relating $E_{a n} / E_{0}$ and $B_{n} / B_{0}$ for the condition that the outermost electrons in the cloud have just reached synchronism.

$$
\begin{aligned}
\frac{E_{a n}}{E_{0}}=\frac{B_{n}}{B_{0}} \frac{r_{c}{ }^{2} / r_{a}{ }^{2}}{\left(B_{n} / B_{0}\right)-\left[1-\left(r_{c}{ }^{2} / r_{a}{ }^{2}\right)\right]} & \\
& \times\left\{2\left[\frac{B_{n}}{B_{0}} \frac{2}{1-\left(r_{c}{ }^{2} / r_{a}{ }^{2}\right)}-1\right]\right. \\
& \left.\times \log \frac{\left\{\left(B_{n} / B_{0}\right)-\left[1-\left(r_{c}{ }^{2} / r_{a}^{2}\right)\right]\right\}^{2}}{r_{c} / r_{a}\left(B_{n} / B_{0}\right)^{\frac{1}{2}}}+1\right\}^{3}
\end{aligned}
$$

${ }^{3}$ See H. W. Welch, Proc. Inst. Radio Engrs. p. 1434 (December, 1950) for experimental confirmation of this expression. 


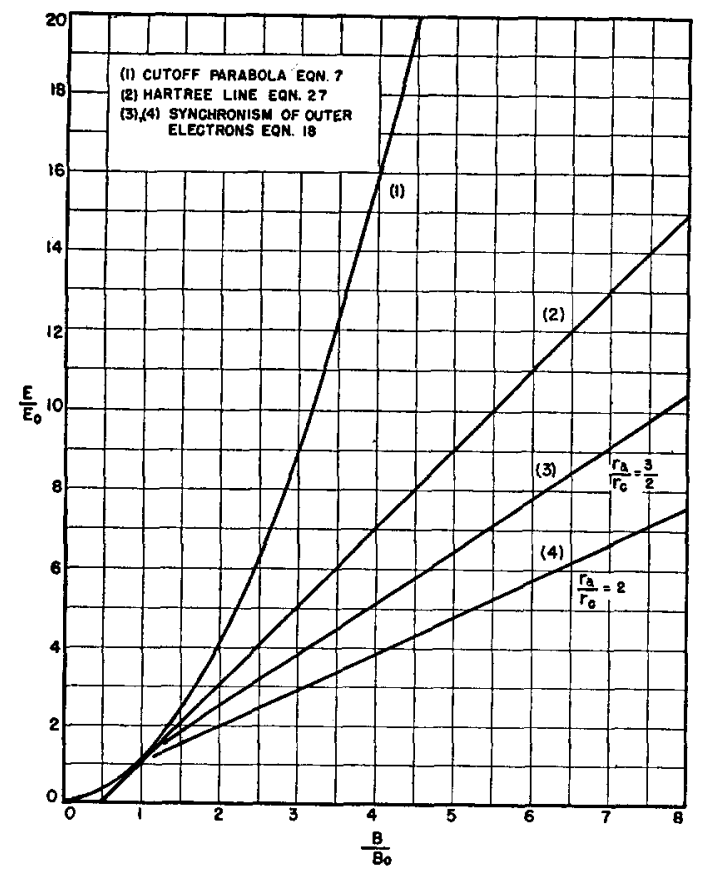

FIG. 3. Anode voltages for cutoff, Hartree, and synchronous boundaries as function of magnetic field.

\section{SYNCHRONISM : CONDITION FOR INITIATION OF OSCILLATION}

It is convenient at this point to discuss an idealized type of space charge behavior which is similar in principle to the case in the static magnetron corresponding to distributions of Eqs. (7)-(9) existing throughout the space charge swarm. It will be assumed that an rf field is present traveling around the interaction space with an angular velocity given by Eq. (12). At the voltage given by Eq. (18) electrons at the outer edge of the swarm formed by the dc fields attain this velocity. Above this voltage the outer electrons are trying to travel faster than the field. The field has tangential components which may either oppose or aid the motion of the electron, depending on the position of the electron relative to the traveling wave. The electrons which take energy from the field will drift inward and eventually give up their excess energy to the cathode. In regions of opposing field the electrons will give up energy to the rf field and be slowed to synchronous velocity. During the process of energy absorption by the field the electrons will drift outward from the edge of the static swarm due to a decrease in the Lorentz force. The potential distribution outside the swarm will thus be altered by presence of the space charge.

Let us now examine the potential distribution in a region where electrons are assumed to be in synchronism. This can be derived quite simply from the original force equation if we make the initial assumption that the angular velocity of the electrons is the synchronous velocity $\omega_{n}$. In this case, Eq. (3a) is written

$$
d v_{r} / d t-r \omega_{n}^{2}=-e / m\left(F_{r}+r \omega_{n} B_{z}\right)
$$

We assume as we did before steady state with no radial motion; i.e., all energy is kinetic energy of rotation. In this case it will be convenient to make the substitution:

We have therefore

$$
\omega_{c}=B e / m=2 \omega_{L} \text {. }
$$

$$
F_{r}=-\partial E / \partial r=(m / e) r \omega_{n}\left(\omega_{n}-\omega_{c}\right) .
$$

This equation can be rewritten

$$
-\frac{\partial}{\partial r}\left(\frac{E}{E_{0}}\right)=2 \frac{r}{r_{a}^{2}}\left(1-\frac{\omega_{c}}{\omega_{n}}\right) .
$$

Integrating this becomes:

$$
-E / E_{0}=\left[1-\left(\omega_{c} / \omega_{n}\right)\right]\left(r^{2} / r_{a}^{2}\right)+C .
$$

In order to evaluate $C$ we will make use of the assumption that all energy is kinetic energy of angular velocity. Thus as the synchronous boundary $r=r_{n}$

and at the anode

$$
e E_{n}=m / 2 \omega_{n}{ }^{2} r_{n}{ }^{2}
$$

$$
e E_{0}=m / 2 \omega_{n}{ }^{2} r_{a}^{2} .
$$

Therefore, dividing one equation by the other, we have at $r=r_{n}$

$$
E_{n} / E_{0}=r_{n}^{2} / r_{a}^{2}
$$

This equation is plotted for comparison with Eq. (17) in Fig. 1. Using this relationship as a boundary condition to evaluate $C$, Eq. (21) becomes

$$
\frac{E}{E_{0}}=-\frac{r^{2}}{r_{a}{ }^{2}}+2 \frac{r_{n}^{2}}{r_{a}{ }^{2}}+\frac{\omega_{c}}{\omega_{n}}\left(\frac{r^{2}}{r_{a}^{2}}-\frac{r_{n}^{2}}{r_{a}{ }^{2}}\right) .
$$

There are two ways of expressing $\omega_{c} / \omega_{n}$ which are useful in simplifying Eq. (23). These are

$$
\frac{\omega_{c}}{\omega_{n}}=\frac{B}{B_{0}} \frac{1}{1-\left(r_{c}^{2} / r_{a}^{2}\right)}
$$

[from the definitions of $\omega_{c}$ in (19) and $B_{0}$ in (14)] and

$$
\omega_{n} / \omega_{c}=\frac{1}{2}\left[1-\left(r_{c}^{2} / r_{n}^{2}\right)\right] .
$$

The latter relationship is based on the assumption that the solution in Eq. (5) holds out to $r_{n}$. Rewrite (23)

$$
\frac{E}{E_{0}}=\frac{\omega_{c}}{\omega_{n}}\left(\frac{r^{2}}{r_{a}{ }^{2}}-\frac{r_{n}{ }^{2}}{r_{a}{ }^{2}}+2 \frac{r_{n}{ }^{2}}{r_{a}{ }^{2}} \frac{\omega_{n}}{\omega_{c}}\right)-\frac{r^{2}}{r_{a}^{2}} .
$$

If we make the substitutions (24) and (25) for the quantity $\omega_{c} / \omega_{n}$ outside the bracket and inside the bracket, respectively, the result becomes

$$
\frac{E}{E_{0}}=2 \frac{B}{B_{0}} \frac{\left(r^{2} / r_{a}^{2}\right)-\left(r_{c}^{2} / r_{a}^{2}\right)}{1-\left(r_{c}^{2} / r_{a}^{2}\right)}-\frac{r^{2}}{r_{a}^{2}} .
$$

This new potential distribution exists throughout the distribution of space charge which forms outside the static magnetron space charge cloud because of electrons 
being slowed to synchronism. If the outermost electrons of this cloud are just capable of reaching the anode the magnetron will draw plate current. The electrons must give up energy to reach this radius; therefore, as soon as the magnetron is capable of drawing current, it will be able to use this energy to generate oscillations. This assumes of course that $\omega_{n}$ corresponds to a frequency at which the magnetron resonator will oscillate. This condition may be imposed upon Eq. (26) and we have, for $r=r_{a}$

$$
E_{a} / E_{0}=\left(2 \dot{B} / B_{0}\right)-1 .
$$

This is a well known relationship obtained in a different way by Hartree ${ }^{4}$ and defining the voltage at which oscillations begin. The assumptions and method used here make clear that the potential of Eq. (27) with the distribution of Eqs. (7) and (26) represent the minimum energy of the individual electron which allows electrons to run in circular orbits extending all the way out to the anode and having synchronous velocity at the anode.

If the anode potential is raised above the value given by Eq. (27) the electrons must have more energy and therefore radial current will exist or the synchronous angular velocity must be increased.

Equations (7), (18), and (27) are plotted for comparison in Fig. 3 in dimensionless variables. It should be noted that the synchronous voltage of $\mathrm{Eq}$. (18) is reached well below the Hartree voltage of Eq. (27). This means that, if a radio frequency is impressed on the anodes the synchronous space charge swarm should start to form well below the Hartree voltage. The synchronous voltage can be observed by measuring the resonant wavelength of a hot magnetron as the anode voltage is raised. When the synchronous voltage is reached a rather abrupt increase in resonant wavelength is observed due to the expansion of the synchronous space charge. ${ }^{5}$

\section{SYNCHRONOUS SPACE CHARGE}

One other equation of some interest can be derived from these relationships. This is the equation for the space charge under the conditions of what we will henceforth call the synchronous space charge distribution. If we apply the poisson equation to Eq. (20) the result is

$$
\rho=-2 \epsilon_{0} m / e \omega_{n}\left(\omega_{c}-\omega_{n}\right) .
$$

The space charge in the synchronous space charge distribution is therefore a constant independent of the radius and dependent only on the angular velocity of the wave, the magnetic field and physical constants.

If the distribution in the portion of the space charge cloud traveling at less than the synchronous velocity $\omega_{n}$ is presumed to be the static magnetron distribution

"D. R. Hartree, "Estimates of Electron Energies, Oscillation Amplitudes, and Efficiencies in a Magnetron Operating under Rotating Space Charge Conditions," C. V. D. Report, Ref. Mag. 11.

${ }_{5}$ Experiments of this type are discussed in reference 3.

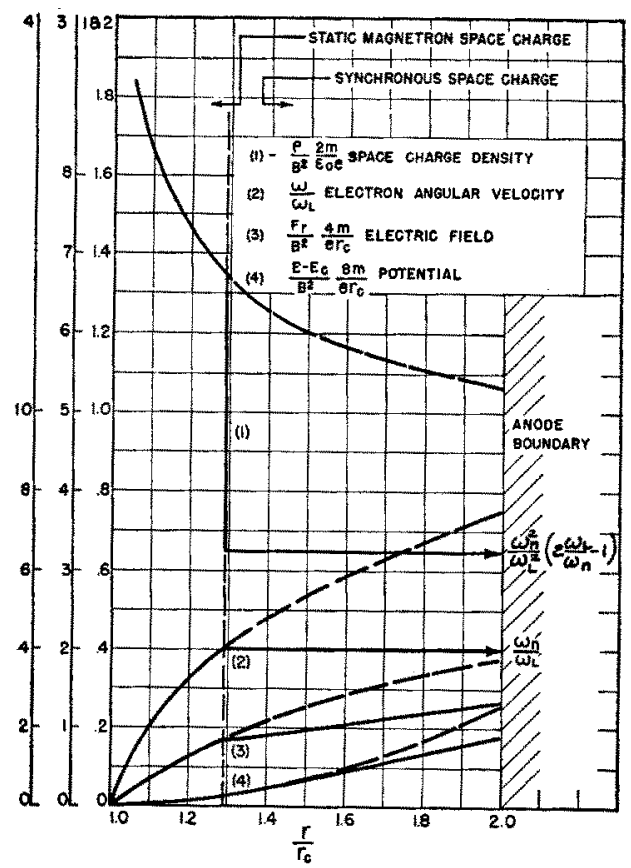

Fyc. 4. Distribution in typical magnetron as synchronous swarm reaches the anode for $\omega_{n} / \omega_{L}=0.4$. Note: dotted curves are continuation of static distribution.

given by Eq. (8); the space charge density at the outside edge of the static magnetron swarm and the inside edge of the synchronous cloud are of different magnitude. Making the substitution

$$
\omega_{n}=\omega_{L}\left[1-\left(r_{c}^{2} / r_{n}^{2}\right)\right]
$$

in the equation for the static magnetron space charge distribution (8), this equation becomes

$$
\rho=-2 \epsilon_{0}(m / e)\left[\left(\omega_{c}^{2} / 2\right)-\omega_{n}\left(\omega_{c}-\omega_{n}\right)\right]
$$

at the boundary between the two distributions. Thus, at the boundary

$$
\frac{\rho_{\text {static }}}{\rho_{\text {synch }}}=\frac{1}{2} \frac{\omega_{c}^{2}}{\omega_{n}\left(\omega_{c}-\omega_{n}\right)}-1 .
$$

Similar algebraic manipulation with the equations for the field and for the potential distribution will show that these quantities are exactly equal at the boundary between the two clouds.

In order to summarize the two types of distributions they are plotted for comparison in Fig. 4. Figure 5 is a pictorial representation of the space charge showing the inner static magnetron swarm and the outer synchronous swarm. Since electrons can only move outward in regions of decelerating rf field the outer swarm is shown as consisting of "spokes" of space charge. This picture of space charge spokes in the oscillating magnetron has been presented by Hartree, Slater, and others and is generally accepted by workers in the magnetron field. Equations (16), (17), (18), and (28) represent relationships which make possible quantitative calculation of synchronous space charge or "spoke" behavior. 


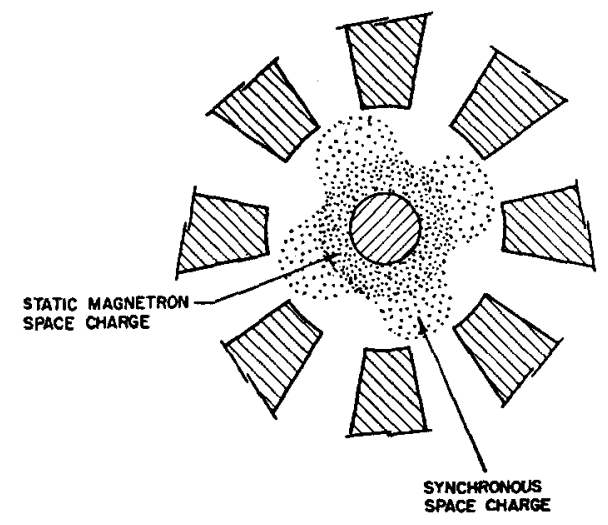

FIG. 5. Pictorial representation of space charge within interaction space.

\section{INITIATION OF ANODE CURRENT; NOISE IN MAGNETRONS 6}

The main purpose in presenting completely the preceding analysis of magnetron synchronous space charge is to make clear the nature of the assumptions which are used and therefore the conditions under which the analysis is true. The resulting values of voltages for the magnetic diode cutoff [Eq. (7) for $r=r_{a}$ ] and voltage for initiation of oscillation [Eq. (27)] have been rightfully criticized for not giving good agreement with experimentally observed results. However, examination of the assumptions shows that both of these voltages are based on the condition that the electron has acquired the minimum amount of kinetic energy of angular velocity required for existence in a stable state at the anode radius. Thus plate current must exist at higher voltages. If current initiates at lower voltages, the electrons must have a smaller minimum kinetic energy and thus smaller angular velocities. If this is the case, they must have been slowed down by some mechanism in their transit to the anode. The only significant mechanism by which the electrons can be decelerated is in a transfer of energy from the dc field to some electromagnetic field. In the case of the initiation of oscillation, it is assumed that electrons have been slowed to synchronous angular velocities by the interaction with the oscillatory electromagnetic field. However, it is experimentally observed that noise voltages exist in preoscillating magnetrons and in static magnetrons operating below cutoff, and it is logical to assume that the production of these voltages is the mechanism by which electrons give up their energy, and therefore reach the anode at lower than predicted values of $\mathrm{dc}$ voltage. Conversely, if noise is produced at a higher level than accounted for by the cathode temperature, it is necessary that anode current be drawn. This is true for the simple reason that, if noise energy is to be produced by

\footnotetext{
6 R. L. Sproull, J. Appl. Phys. 18, 314-320 (1947) : V. Mayper, "Noise Generation in Pre-Oscillating Magnetron," Quarterly Progress Report, M.I.T. Research Laboratory of Electronics, 4-6 (January 15, 1948).
}

the system, then energy must be given the system by the power supply. The noise probably originates in the cathode as thermal noise, etc., and is amplified by the process of continuous interchange of energy between the $\mathrm{dc}$ field and the noise field through the medium of the electron swarm. On the basis of these ideas, it is suggested that experiments designed to cast light on the origins of noise produced by magnetrons should be made on non-oscillating structures and correlated carefully with the dc current drawn by the magnetron. It is also possible that an amplifier could be constructed employing the principle of interaction between layers of magnetron space charge traveling at different angular velocities similar to the already constructed electronwave tube. ${ }^{7}$

\section{OSCILLATION; ELECTRONIC EFFICIENCY}

The definition of the voltage for initiation of oscillation given by $\mathrm{Eq}$. (27) has a similarity in principle to the magnetic diode cutoff voltage in that the electrons have the minimum kinetic energy of angular velocity required for their existence at the anode. It is noteworthy that the kinetic energy of the electrons at the anode is in general less than the energy they have acquired, the difference being the energy supposedly delivered to a rotating electromagnetic field with which they are synchronous. Thus it is possible to calculate the maximum electronic efficiency which is obtainable under the given conditions of voltage and tube geometry. If $E_{a}$ is the anode voltage at which anode current is drawn, representing energy delivered to the electron, and $E_{0}$ is the voltage defined by Eq. (15), representing the kinetic energy of the synchronous electron, then maximum electronic efficiency is given by

$$
\eta_{e}=\left(E / E_{0}-1\right) / E / E_{0} .
$$

Thus for interaction efficiency greater than 80 percent, $E / E_{0}$ must be greater than 5 . This places certain restrictions on $r_{a}$ and $n$ and determines $B / B_{0}$ through Eq. (27).

A further consideration which must be included in an exact analysis of the oscillating magnetron is the effect of loading. The fact that an electron may exist at the anode and thus permit oscillation to begin, does not imply that the negative conductance which the space charge provides will meet the conditions imposed by the load. Thus, experimentally it is observed that voltage for initiation of oscillation depends on the loading in addition to the other factors. The maximum electronic efficiency given by Eq. (30) is observed in some cases, but can only exist under optimum load conditions. No analysis has been presented, to our knowledge, which predicts quantitatively the effect of loading conditions on efficiency, although experimental data on a particular tube enables one to make semiquantitative predictions.

${ }^{7}$ A. V. Haeff, Proc. Inst. Radio Engrs. (January, 1949). 